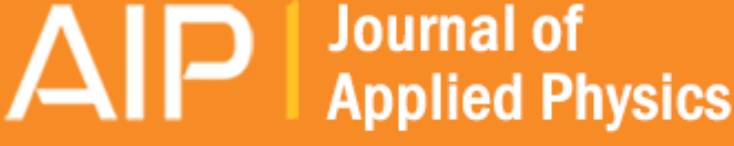

\section{Plasmon-induced photoexcitation of "hot" electrons and "hot" holes in amorphous silicon photosensitive devices containing silver nanoparticles}

Etienne Antoine Moulin, Ulrich Wilhelm Paetzold, Bart Elger Pieters, Wilfried Reetz, and Reinhard Carius

Citation: Journal of Applied Physics 113, 144501 (2013); doi: 10.1063/1.4795509

View online: http://dx.doi.org/10.1063/1.4795509

View Table of Contents: http://scitation.aip.org/content/aip/journal/jap/113/14?ver=pdfcov

Published by the AIP Publishing

\section{Articles you may be interested in}

Erratum: "Plasmon-induced photoexcitation of "hot" electrons and "hot" holes in amorphous silicon photosensitive devices containing silver nanoparticles" [J. Appl. Phys. 113, 144501 (2013)]

J. Appl. Phys. 114, 089901 (2013); 10.1063/1.4818359

Largely enhanced photocurrent via gap-mode plasmon resonance by a nanocomposite layer of silver nanoparticles and porphyrin derivatives fabricated on an electrode

Appl. Phys. Lett. 101, 063103 (2012); 10.1063/1.4742870

Plasmon enhanced resonant defect absorption in thin a-Si:H n-i-p devices

Appl. Phys. Lett. 100, 253907 (2012); 10.1063/1.4730432

Photoresponse enhancement in the near infrared wavelength range of ultrathin amorphous silicon photosensitive devices by integration of silver nanoparticles

Appl. Phys. Lett. 95, 033505 (2009); 10.1063/1.3157264

Subpicosecond hot carrier cooling in amorphous silicon

J. Appl. Phys. 84, 4984 (1998); 10.1063/1.368744

You don't

still use this

cell phone

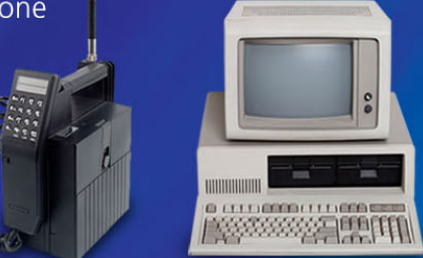

Why are you still using an AFM designed in the 80 's?
It is time to upgrade your AFM

Minimum $\$ 20,000$ trade-in discount for purchases before August 31st

Asylum Research is today's technology leader in AFM 


\title{
Plasmon-induced photoexcitation of "hot" electrons and "hot" holes in amorphous silicon photosensitive devices containing silver nanoparticles
}

\author{
Etienne Antoine Moulin, ${ }^{\text {a) }}$ Ulrich Wilhelm Paetzold, Bart Elger Pieters, Wilfried Reetz, \\ and Reinhard Carius \\ IEK5-Photovoltaik, Forschungszentrum Jülich GmbH, 52425 Jülich, Germany
}

(Received 24 October 2012; accepted 2 March 2013; published online 8 April 2013)

\begin{abstract}
We report on a plasmon-induced photocurrent in photosensitive devices based on hydrogenated amorphous silicon (a-Si:H) containing silver nanoparticles (NPs). The photocurrent is measured in a spectral region corresponding to optical transitions below the band gap of a-Si:H. Photoexcitation of "hot" electrons in the NPs or in defect states present in the vicinity of the NPs, resulting from plasmon decay in the NPs, is often cited as being responsible for this effect. In this study, we demonstrate that plasmon induced photogeneration of "hot" holes is also able to contribute to a photocurrent. A bifacial symmetrical transparent device was prepared in order to compare the internal quantum efficiency of both processes, the first based on the photogeneration of "hot" electrons and the second based on the photogeneration of "hot" holes. (C) 2013 American Institute of Physics. [http://dx.doi.org/10.1063/1.4795509]
\end{abstract}

\section{INTRODUCTION}

Several studies have exploited the optical properties of metal nanoparticles (NPs) for improving the performance of photoactive devices such as photodetectors, light-emitting diodes, and solar cells. ${ }^{1-8}$ Depending on the size, shape, and surrounding material of a metal NP, coherent collective oscillations of the quasi-free electrons of a metal NP can be excited by an incident electromagnetic wave of a specific wavelength. These collective excitations of electrons are referred to as localized surface plasmon polaritons (LSPPs). ${ }^{9,10}$ The radiative decay of these oscillations into propagating electromagnetic waves is observed as scattering of incident light by the NPs. The scattering properties of large NPs (with diameter $\varnothing>40 \mathrm{~nm})^{9-11}$ have often been used to increase the optical path length within solar cells and consequently enhance the short-circuit photocurrent density. ${ }^{12-17}$ Plasmonic resonances in smaller metal NPs $(\varnothing<40 \mathrm{~nm})$ lead to a particularly strong enhancement of the electric field intensity in the NPs and in their direct vicinity. The increased light absorption induced by this enhanced local electric field has also been shown to play a beneficial role in photoactive device applications.

We found, for example, that ultra-thin photosensitive devices based on hydrogenated amorphous silicon (a-Si:H) containing small Ag NPs (with $\varnothing$ around $15 \mathrm{~nm}$ ) are able to generate a relatively high photocurrent in a spectral range corresponding to optical transitions below the band gap of a-Si:H. ${ }^{18,19}$ The device configuration (denoted as NPs-i-n) used for achieving this effect is illustrated in Fig. 1. In a-Si:H, a photocurrent at sub-band-gap wavelengths can also be measured without NPs, due to excitation of carriers from defects present in the band gap of the material. However, in the presence of NPs, the measured photocurrent is much

\footnotetext{
a) Author to whom correspondence should be addressed. Electronic mail: e.moulin@fz-juelich.de. Telephone: +49 2461 61-2069. Fax: +49 2461 61-3735.
}

larger than that expected from such a process. The existence of a photocurrent in the near infrared (NIR) region is correlated with a strong increase in light absorption in the NPs at wavelengths close to the NPs LSPP resonance. On one hand, damping of a LSPP in a NP can dissipate photon energy into heat. On the other hand, the energy arising from plasmon decay can also be transferred to (i) a single electron of the $\mathrm{NP}^{20-24}$ or to (ii) an electron present in the vicinity of the NP (e.g., in a defect state located in the band gap of the semiconductor). As shown in Fig. 1, for sufficiently high energy, this so-called "hot" electron can be injected over the Schottky barrier formed by the NP-semiconductor interface (in case (i) $)^{5-8,25}$ or can directly reach the conduction band of the semiconductor (in case (ii)). This electron can then be transported by drift towards the opposite electrode of the device, thereby providing a photocurrent (Fig. 1).

A photocurrent was also measured in the NIR wavelength region for NPs-i- $p$ photosensitive devices with $\mathrm{Ag}$ NPs. ${ }^{18}$ As the transport of electrons from the NPs towards the front contact should be effectively prohibited by replacing the $\mathrm{n}$-doped layer by a p-doped layer, uncertainties remain regarding the carrier transport in this device. Since a very thin absorber layer of $20 \mathrm{~nm}$ has been applied in the devices discussed in Ref. 18, the current transport might be based on trap-assisted tunnelling of electrons from the p-doped layer towards the NPs. The positive effect of the NPs in the NPs-i-p device configuration might, therefore, be observable only in the particular case where an ultra-thin i-layer is employed.

To further investigate the effect of NPs on photosensitive device behavior with consideration for the above possibility, thicker devices with an i-layer thickness of $150 \mathrm{~nm}$ are employed in the present study. Optical and electrical characterizations of these devices are used to provide insight into the possible photogeneration and transport mechanisms responsible for the photocurrent in the NIR wavelength region. In addition to the LSPP-induced photoexcitation of "hot" electrons, a 

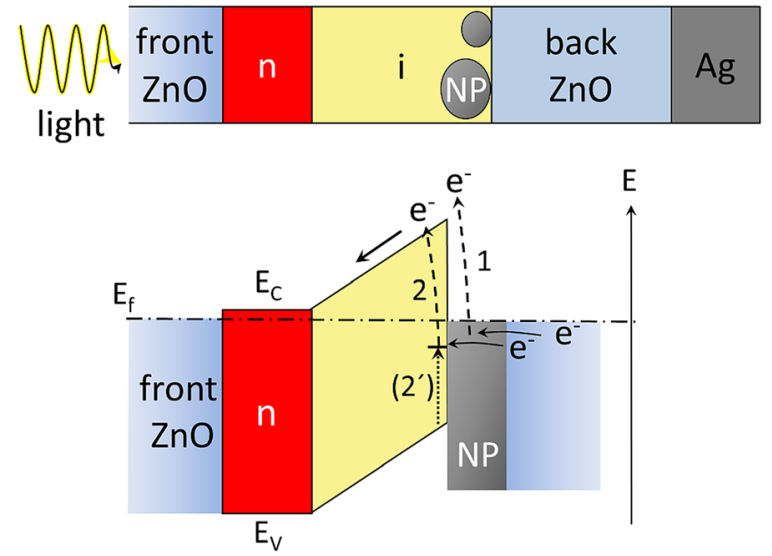

\section{E}

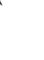


deposited through a mask, on half of the substrate only, so that devices without NPs can be used as references.

The devices without NPs (referred to as i-n and i-p) exhibit an absorptance larger than $60 \%$ between $330 \mathrm{~nm}$ and $600 \mathrm{~nm}$, reaching a maximum of nearly $100 \%$ at $550 \mathrm{~nm}$ (Fig. 3(a), squares). Between $550 \mathrm{~nm}$ and $700 \mathrm{~nm}$, the absorptance decreases abruptly. For $\lambda>700 \mathrm{~nm}$, values below $10 \%$ are measured. For $\lambda>900 \mathrm{~nm}$, the absorptance is negligible. This rapid decrease is explained by the low absorption coefficient of a-Si:H in the NIR spectrum. The integration of NPs leads to a significant increase in the absorptance, for both devices, at $\lambda>600 \mathrm{~nm}$ (Fig. 3(a), circles). The absorptance reaches almost $100 \%$ at $800 \mathrm{~nm}$ and $900 \mathrm{~nm}$ for the NPs-i-p and NPs-i-n devices, respectively, before declining at longer wavelengths. The slight differences found for both devices probably result from the use of different front layers, namely the $\mathrm{p}$ - and $\mathrm{n}$-doped layers, and a remaining inhomogeneity in the layer thicknesses of the co-deposited devices. The broad maximum in absorptance between $700 \mathrm{~nm}$ and $1100 \mathrm{~nm}$ associated with the LSPP resonance is presumably caused by (i) the broad distribution of shapes and sizes of the $\mathrm{NPs},{ }^{9,10,28,29}$ (ii) the interaction between the NPs, ${ }^{9}$ and (iii) the presence of the silver back reflector, which leads to interferences that partly mask the LSPP resonance.

At $\lambda<580 \mathrm{~nm}$, the EQE of the i-p device is higher than the EQE of the i-n device (cf. Fig. 3(b)). This is due to a lower parasitic absorptance in the front a-SiC:H p-layer than in the a-Si:H n-layer. In accordance with the absorptance data, the EQEs of both devices without NPs drop between

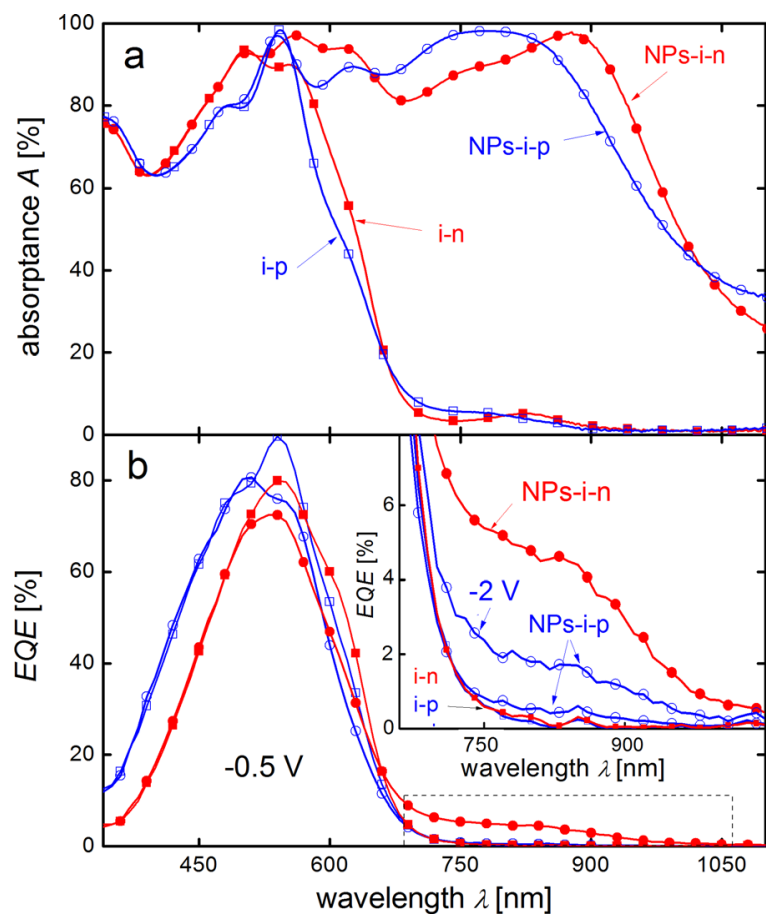

FIG. 3. Absorptance as a function of wavelength (a) and external quantum efficiency at $-0.5 \mathrm{~V}$ (b) of i-n and i-p photosensitive devices (filled and open symbols, respectively) with (circles) and without (squares) nanoparticles. The inset in (b) shows an enlargement of the EQE in the long wavelength region (delimited by the dashed box). Please note that the EQE data of the NPs-i-p device measured at $-2 \mathrm{~V}$ is added in the inset of (b).
$550 \mathrm{~nm}$ and $700 \mathrm{~nm}$ and reach a negligible value at $\lambda>$ $750 \mathrm{~nm}$. The EQEs are not modified in the short-wavelength range by incorporating the NPs. This indicates that the extraction and collection of the generated carriers are not noticeably altered by the presence of the NPs. At $-0.5 \mathrm{~V}$, the EQE of the NPs-i-n device has already reached its saturation value, confirming the satisfying extraction and collection of carriers under moderate bias voltage. Since the photocurrent in the NIR wavelength region is not limited by the carrier collection, a definitive number can be given regarding the internal quantum efficiency (IQE) of the LSPP-induced photogeneration mechanism of the NPs-i-n device. Considering that most of the light in the NIR region is absorbed by the NPs (or by the first nanometers of a-Si:H surrounding the NPs), an IQE of around $5 \%$ and $3 \%$ is found at $800 \mathrm{~nm}$ and $900 \mathrm{~nm}$, respectively.

The above result can be explained with the model based on the LSPP-induced photogeneration of "hot" electrons in the NPs (or from defect states in their vicinity) and the transport of these electrons towards the front contact (cf. Fig. 1). However, if the n-doped layer is replaced by a p-doped layer, we would expect the transport of electrons from the NPs towards the front contact to be effectively prohibited. Interestingly, though, the NPs-i-p device also shows a photocurrent in the NIR wavelength region. For a bias voltage of $-0.5 \mathrm{~V}$, the EQE signal is negligible but at a bias voltage of $-2 \mathrm{~V}$, the EQE reaches approximately $2 \%$ (Fig. 3(b), inset, open circles). As the transport mechanism of trap-assisted tunnelling of electrons from the p-doped contact towards the NPs can be excluded owing to the thickness of the absorber i-layer used for the devices under study, this result indicates the existence of a mechanism based on the photogeneration and transport of holes in this device. This underlines the reciprocity of the mechanisms involved in the photogeneration process and carrier transport for both the NPs-i-n and the NPs-i-p devices. On the basis of the model illustrated in Fig. 1, a model is shown in Fig. 4 that depicts the LSPP-induced photogeneration of "hot" holes in the NPs (process 1) —or from states in their vicinity (process 2) -and the transport of these holes towards the p-doped contact.

As the photocurrent of the NPs-i-p device does not saturate in the NIR wavelength region with increasing negative bias, no definitive number can be given regarding the IQE of the associated photogeneration process (illustrated in Fig. 4). The photocurrent might suffer from a poor extraction and collection of holes, even at a reverse bias of $-2 \mathrm{~V}$, because of the following reasons: (i) the low mobility of holes in a-Si:H, ${ }^{30}$ (ii) the existence of a barrier hindering their transport through the i-layer, and/or (iii) the presence of defects at the NPs/a-Si:H interface resulting in an enhanced surface recombination.

To investigate this, a symmetrical transparent bifacial device has been prepared. With this approach, each photogeneration process-based on the photoexcitation of electrons (see Fig. 1) as well as holes (see Fig. 4) — and associated transport mechanism can be addressed alternatively by simply inverting the polarity of the applied bias voltage. Moreover, as the same device can be employed for activating both transport 

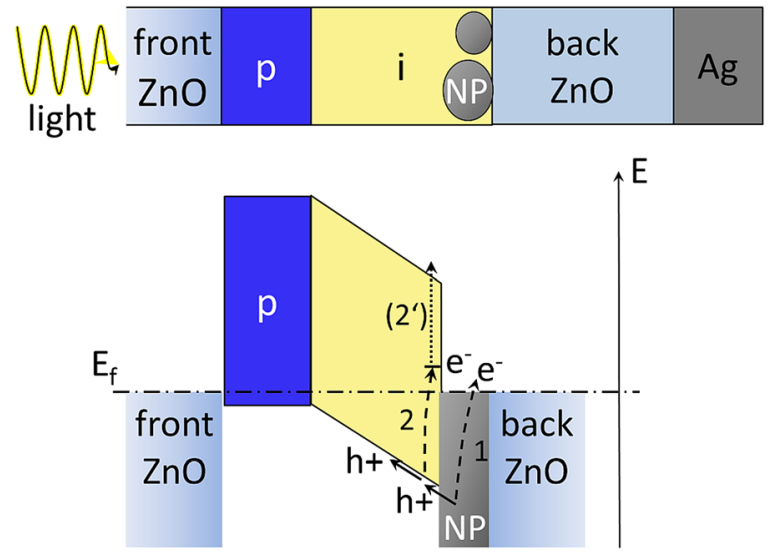

FIG. 4. Schematic drawing of the NPs-i-p device configuration (top) and corresponding schematic band diagram (bottom). Process 1 describes the LSPPinduced photoexcitation of a "hot" hole-(electron pair) in the NP. Process 2 depicts the LSPP-induced photoexcitation of a "hot" hole-(electron pair) in the immediate vicinity of the NP. In process 2 , the electron is excited to a defect state within the band gap of a-Si:H above the Fermi level. The electron might reach the conduction band of a-Si:H by an additional thermal activation step (process $2^{\prime}$ ). ${ }^{26}$ The transport of the resulting holes towards the front $\mathrm{p}$ doped contact is illustrated as well.

processes, discrepancies in the optoelectrical device properties arising from inhomogeneity problems can be suppressed.

\section{B. Symmetrical transparent bifacial device illuminated alternatively from both sides}

Fig. 5 (top) shows an illustration of the symmetrical transparent bifacial device and the corresponding schematic band diagrams according to the polarity of the applied bias voltage. For a positive bias, electrons are transported towards the front contact and holes towards the back contact. At negative bias, the carriers flow in the opposite direction. The transport of the charges for a positive bias is thus similar to that of the NPs-i-n device configuration. The carrier transport under negative bias is the same as for the NPs-i-p device configuration.

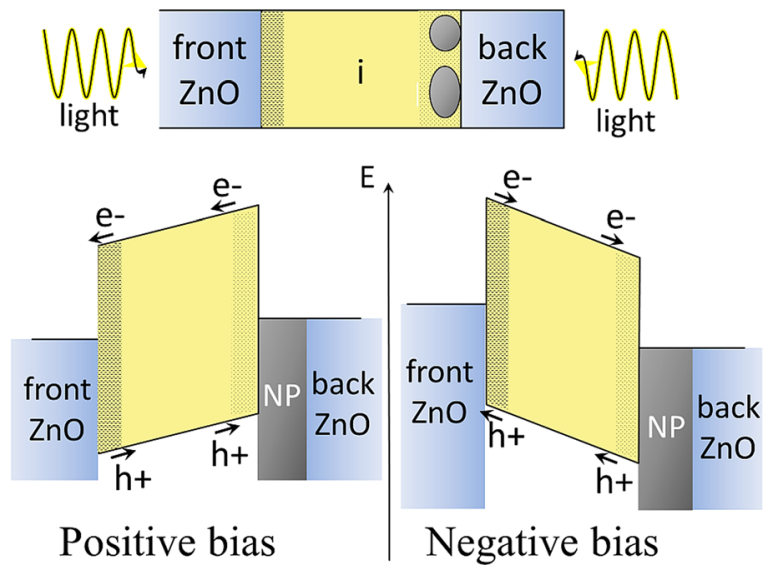

FIG. 5. Schematic drawing of the transparent bifacial NPs-i device (top) and corresponding simplified band diagram according to the polarity of the applied bias voltage (bottom). Depending on the illumination side (front/back side), the hatched/dotted area represents the portion of a-Si:H, where light of short wavelength $\lambda(\lambda<450 \mathrm{~nm})$ is absorbed, leading to the photogeneration of electron-hole pairs. The arrows indicate the direction in which the electrons and holes are transported.

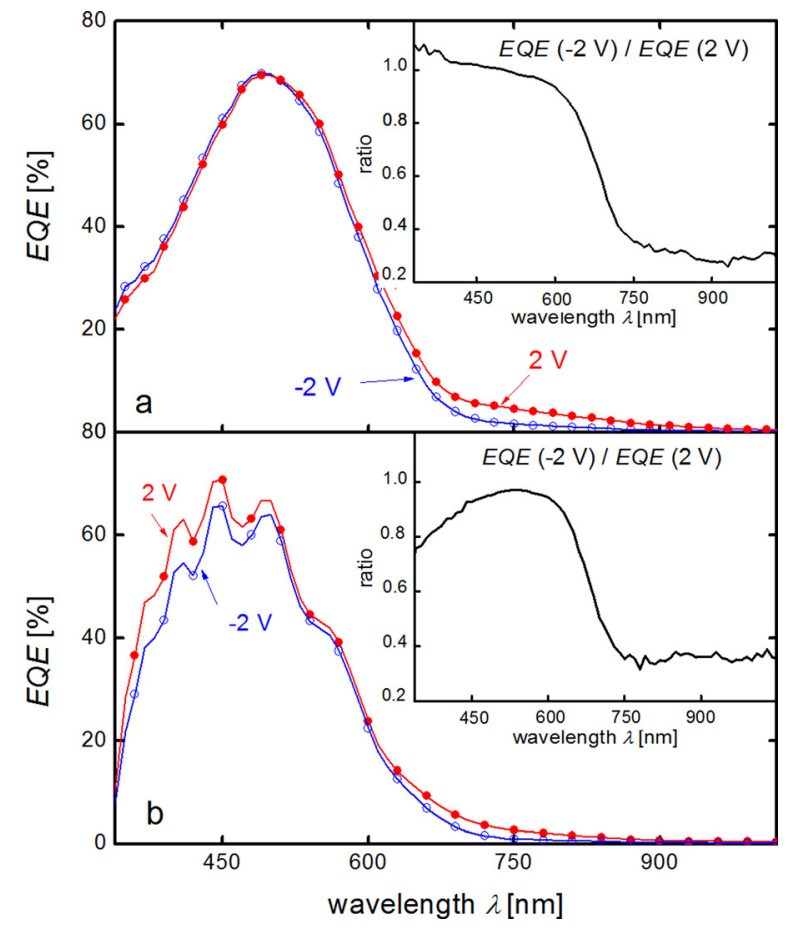

FIG. 6. EQE for an illumination from the front side (a) and for an illumination from the rear side (b). The insets show the ratios between the EQE at negative and positive bias voltages.

The EQE of the device illuminated from the front side for positive and negative bias polarities is presented in Fig. 6(a). In the short wavelength range (for $\lambda<450 \mathrm{~nm}$ ), the EQEs at $-2 \mathrm{~V}$ and $2 \mathrm{~V}$ have reached their maximum value, indicating that nearly all carriers generated in the topmost portion of the device (cf. Fig. 5, hatched area) are efficiently collected. In contrast, at $\lambda>750 \mathrm{~nm}$, the device exhibits a significantly lower EQE at negative bias. In this spectral range, the ratio between the EQE measured at $-2 \mathrm{~V}$ and $2 \mathrm{~V}$ is equal to approximately 0.3 (cf. inset in Fig. 6(a)). This is in good agreement with the difference found in Sec. III A between the NPs-i-n and NPs-i-p configurations. This discrepancy might result from the poor extraction and collection of holes generated at the LSPP resonance within the NPs (process 1 ) and/or in their immediate vicinity (process 2). To verify this assumption, the device has been illuminated from the rear side. The corresponding EQEs are shown in Fig. 6(b).

For $\lambda<450 \mathrm{~nm}$, the EQE increases rapidly with elevating bias and already reaches its maximum saturation value at moderate positive bias voltage (not shown here). At negative voltage, this increase is significantly slower (not shown here). In this case, holes generated in the bottommost portion of the device (cf. Fig. 5, dotted area) have to (i) leave the presumably defect-rich region adjacent to the NPs/a-Si:H interface and (ii) traverse the whole i-layer before arriving at the front contact. In the short wavelength region, the EQE measured at $-2 \mathrm{~V}$ nearly reaches $80 \%$ of the EQE measured at $2 \mathrm{~V}$ (cf. inset in Fig. 6(b)), indicating that $80 \%$ of the holes generated in the bottommost portion of the device (i.e., in the vicinity of the NPs) reach the front contact. If we assume that $100 \%$ of the photogenerated electrons resulting from the LSPP effect are effectively collected at $2 \mathrm{~V}$ and that the LSPP-induced photogeneration of holes is as efficient as that 
of electrons (i.e., $100 \%$ of generated holes), we would expect that $80 \%$ of these holes would be collected at $-2 \mathrm{~V}$. An EQE $(-2 \mathrm{~V} / 2 \mathrm{~V})$ ratio of $80 \%$ would thus be measured in the NIR wavelength range. However, measurements in this spectral region show a ratio of only 0.35 (cf. inset in Fig. 6(b)).

This discrepancy could be explained by (i) a higher recombination rate of holes due to a shorter lifetime of the LSPP-induced holes photoexcited in the NPs (or in their direct vicinity) and (ii) a higher energy barrier for holes than for electrons. In the NIR wavelength, the maximal excitation energy of the LSPP-induced carriers is approximately $1.7 \mathrm{eV}$. In the literature, it was shown that in this energy range electrons and holes in $\mathrm{Ag}$ having similar excitation energies with respect to the Fermi level possess almost similar lifetimes. ${ }^{31}$ As the density of states in Ag is nearly constant in this energy range, ${ }^{31}$ we expect that electrons and holes excited in the metal statistically possess the same excess energy with respect to the Fermi level. Therefore, it is also expected that both types of carriers photoexcited in $\mathrm{Ag}$ possess, on the average, the same lifetime. Consequently, argument (i), stating that a shorter lifetime of holes in the NPs is responsible for the observed discrepancy in EQE in the NIR wavelength region, does not hold. Nevertheless, in the case the LSPP-induced carriers would be generated in the direct vicinity of the NPs, it is not straightforward to predict whether the lifetime of holes and electrons is identical, due the high defect density at the NPs/semiconductor interface. Although a final conclusion cannot be made on the basis of the present results, explanation (ii) seems more likely as the Fermi level in the intrinsic a-Si:H is slightly above midgap, making a-Si:H-i an n-type material. A lower energy would thus be required for electrons to overcome the barrier at the NPs/semiconductor interface than for holes.

\section{CONCLUSIONS}

The effect of small silver NPs on the optoelectrical properties of a-Si:H-based photosensitive devices has been investigated. The devices with NPs show a strong absorption in the near infrared wavelength region, reaching nearly $100 \%$. This strong absorption is associated with a detectable photocurrent in the same spectral range. A maximal internal quantum efficiency of the photogeneration process reaching nearly $5 \%$ has been measured, making it interesting for detector applications. The existence of a photocurrent at energies below the semiconductor band gap is explained in terms of photoexcitation of "hot" electron-hole pairs, inside the NPs and/or in their immediate vicinity, arising from plasmon decay. While "hot" electrons are often seen as being responsible for the existence of a photocurrent in the near infrared wavelength region, we have shown that "hot" holes also contribute to a detectable photocurrent in this spectral range. Measurements performed on an a-Si:H-based symmetrical bifacial device suggest that "hot" electrons contribute more significantly to the photocurrent than "hot" holes.

\section{ACKNOWLEDGMENTS}

We thankfully acknowledge Hans Peter Bochem, Carsten Grates, Joachim Kirchhoff, Tsvetelina Merdzhanova, Andreas Mück, Thomas Müller, Hilde Siekmann, and Janine Worbs for technical support and Karsten Bittkau, Charmaine Chia, Kaining Ding, Maurice Nuys, Vladimir Smirnov for their insight and input. This work was supported by the German Federal Ministry of Education and Research under Contract No. 03SF0354D.

${ }^{1}$ H. A. Atwater and A. Polman, Nature Mater. 9, 205 (2010).

${ }^{2}$ N. Yu, J. Fan, Q. J. Wang, C. Pflügl, L. Diehl, T. Edamura, M. Yamanishi, H. Kann, and F. Capasso, Nat. Photonics 2, 564 (2008).

${ }^{3}$ L. Tang, S. E. Kocabas, S. Latif, A. K. Okyay, D.-S. Ly-Gagnon, K. C. Saraswat, and D. B. Miller, Nat. Photonics 2, 226 (2008).

${ }^{4}$ T. Ishi, J. Fujikata, K. Makita, T. Baba, and K. Ohashi, Jpn. J. Appl. Phys., Part 2 44, L364 (2005).

${ }^{5}$ M. Wesphalen, U. Kreibig, J. Rostalski, H. Luth, and D. Meissner, Sol. Energy Mater. Sol. Cells 61, 97 (2000).

${ }^{6}$ O. Stenzel, A. Stendal, K. Voigtsberger, and C. von Borczyskowski, Sol. Energy Mater. Sol. Cells 37, 337 (1995).

${ }^{7}$ C. Wen, K. Ishikawa, M. Kishima, and K. Yamada, Sol. Energy Mater. Sol. Cells 61, 339 (2000).

${ }^{8}$ M. W. Knight, H. Sobhani, P. Nordlander, and N. J. Halas, Science 332, 702 (2011).

${ }^{9}$ U. Kreibig and M. Vollmer, Optical Properties of Metal Clusters (Springer, New York, 1995).

${ }^{10}$ S. A. Maier, Plasmonics: Fundamentals and Applications (Springer, New York, 2007).

${ }^{11}$ C. F. Bohren and D. R. Huffman, Absorption and Scattering of Light by Small Particles (Wiley, Weinheim, 2004).

${ }^{12}$ D. M. Schaadt, B. Feng, and E. T. Yu, Appl. Phys. Lett. 86, 063106 (2005).

${ }^{13}$ D. Derkacs, S. H. Lim, P. Matheu, W. Mar, and E. T. Yu, Appl. Phys. Lett. 89, 093103 (2006).

${ }^{14}$ S. Pillai, K. R. Catchpole, T. Trupke, and M. A. Green, J. Appl. Phys. 101, 093105 (2007).

${ }^{15}$ E. Moulin, J. Sukmanowski, P. Luo, R. Carius, F. X. Royer, and H. Stiebig, J. Non-Cryst. Solids 354, 2488 (2008).

${ }^{16}$ E. Moulin, J. Sukmanowski, M. Schulte, A. Gordijn, F. X. Royer, and H. Stiebig, Thin Solid Films 516, 6813 (2008).

${ }^{17}$ K. R. Catchpole and A. Polman, Opt. Express 16, 21793 (2008).

${ }^{18}$ E. Moulin, P. Luo, B. Pieters, J. Sukmanowski, J. Kirchhoff, W. Reetz, T. Müller, R. Carius, F.-X. Royer, and H. Stiebig, Appl. Phys. Lett. 95, 033505 (2009).

${ }^{19}$ P. Q. Luo, E. Moulin, J. Sukmanowski, F.-X. Royer, X. M. Dou, and H. Stiebig, Thin Solid Films 517(23), 6256 (2009).

${ }^{20}$ J. G. Endriz and W. E. Spicer, Phys. Rev. Lett. 24, 64 (1970).

${ }^{21}$ T. Inagaki, K. Kagami, and E. T. Arakawa, Phys. Rev. B 24, 3644 (1981)

${ }^{22}$ T. Inagaki, K. Kagami, and E. T. Arakawa, Appl. Opt. 21, 949 (1982).

${ }^{23}$ J. Hofmann and W. Steinmann, Phys. Status Solidi 30, K53 (1968).

${ }^{24}$ J. Lehmann, M. Merschdorf, W. Pfeiffer, A. Thon, S. Voll, and G. Gerber, Phys. Rev. Lett. 85, 2921 (2000).

${ }^{25}$ C. Daboo, M. J. Baird, H. P. Hughes, N. Apsley, and T. Emeny, Thin Solid Films 201, 9 (1991).

${ }^{26}$ F. Lükermann, U. Heinzmann, and H. Stiebig, Appl. Phys. Lett. 100, 253907 (2012).

${ }^{27}$ B. Rech, T. Repmann, M. N. van den Donker, M. Berginski, T. Kilper, J. Hüpkes, S. Calnan, H. Stiebig, and S. Wieder, Thin Solid Films 511-512, 548 (2006).

${ }^{28}$ C. Sönnichsen, T. Franzl, T. Wilk, G. von Plessen, and J. Feldmann, New J. Phys. 4, 93 (2002).

${ }^{29}$ C. Sönnichsen, S. Geier, N. E. Hecker, G. von Plessen, J. Feldmann, H. Ditlbacher, B. Lamprecht, J. R. Krenn, F. R. Aussenegg, V. Z.-H. Chan, J. P. Spatz, and M. Moeller, Appl. Phys. Lett. 77, 2949 (2000).

${ }^{30}$ S. Reynolds, J. Phys.: Conf. Ser. 253(1), 012002 (2010).

${ }^{31}$ V. P. Zhukov, F. Aryasetiawan, E. V. Chulkov, I. G. de Gurtubay, and P. M. Echenique, Phys. Rev. B 64, 195122 (2001). 